\title{
Covid-19: Efeitos da pandemia no surgimento de quadros psiquiátricos entre enfermeiras de Unidade de Terapia Intensiva
}

COVID-19: Effects of the pandemic on the emergence of psychiatric conditions among intensive

\section{Care Unit Nurses}

COVID-19: Efectos de la pandemia en la aparición de condiciones psiquiátricas entre enfermeras de Unidades de Cuidados Intensivos

Maria Jussara Medeiros Nunes ORCID: https://orcid.org/0000-0002-3324-0081 Universidade do Estado do Rio Grande do Norte, Brasil

E-mail: mariajussara@alu.uern.br

Jean Carlos Souza Silva

ORCID: https://orcid.org/0000-0001-8344-5512 Universidade do Estado do Rio Grande do Norte, Brasil

E-mail: jeancbiologia@gmail.com

Antonio William do Nascimento Fernandes

ORCID: https://orcid.org/0000-0001-7925-4887 Universidade do Estado do Rio Grande do Norte, Brasil E-mail: williamfr_g3@hotmail.com

Danielle Christina Lino Leal

ORCID: https://orcid.org/0000-0002-1048-3435 Universidade do Estado do Rio Grande do Norte, Brasil E-mail: danielleleal@alu.uern.br

Isabela Pinheiro Cavalcanti Lima ORCID: https://orcid.org/0000-0002-7681-9675 Universidade do Estado do Rio Grande do Norte, Brasil E-mail: belapc1@yahoo.com.br

\begin{abstract}
Resumo
Introdução: Os enfermeiros da linha de frente enfrentam uma enorme carga de trabalho, fadiga de longo prazo, ameaça de infecção e frustração com a morte de pacientes de quem cuidam. Objetivo: Investigar as atuais evidências sobre o surgimento de quadros psiquiátricos em enfermeiros de Unidades de Terapia Intensiva atuantes durante a pandemia da Covid-19. Metodologia: Estudo de revisão integrativa com busca sistemática, realizada nas bases de dados PubMed, Embase, Cochrane Wiley, e Biblioteca Virtual em Saúde (BVS). Os critérios de inclusão foram: (1) artigos eletrônicos, (2) disponíveis na íntegra, (3) nos idiomas português, inglês e/ou espanhol. Foram excluídos resumos, carta ao editor e estudos que não apresentaram relação com a temática. Resultados: Após aplicação dos critérios de elegibilidade, foram incluídos no estudo 21 artigos. Os principais fatores de risco encontrados foram:
\end{abstract}


Trabalho em cuidados críticos voltados para pacientes com Covid-19, Uso de equipamentos de biossegurança, Infraestrutura hospitalar; Fornecimento inadequado de Equipamentos de Proteção Individual, Idade, gênero, estado civil, especialidade e proximidade com os pacientes Covid-19; Impossibilidade de atender às necessidades psicosocioemocionais dos pacientes e familiares; Dificuldade de externalizar suas emoções; Falta de experiência profissional e Medo de contrair o vírus e infectar outras pessoas. Os principais fatores de risco para os desfechos citados acima foram: Exaustão emocional; estresse psicológico; ansiedade; insônia; medo; raiva e frustração. Conclusões: Os enfermeiros que atuam na UTI durante a pandemia da Covid-19apresentaram quadros de alterações psiquiátricas, tendo como principais fatores causais o estresse emocional, gerado por inadequações estruturais e organizacionais nas instituições de saúde.

Palavras-chave: SARS-CoV-2; Unidades de Terapia Intensiva; Atenção à saúde; Cuidados de enfermagem; Saúde mental.

\begin{abstract}
Introduction: Frontline nurses face an enormous workload, long-term fatigue, threat of infection, and frustration with the death of patients they care for. Objective: To investigate the current evidence on the emergence of psychiatric conditions in nurses working in Intensive Care Units during the Covid-19 pandemic. Methodology: Integrative review study with systematic search, performed in PubMed, Embase, Cochrane Wiley, and Virtual Health Library (VHL) databases. Inclusion criteria were: (1) electronic articles, (2) available in full, (3) in Portuguese, English and/or Spanish. Abstracts, letters to the editor and studies that were not related to the theme were excluded. Results: After applying the eligibility criteria, 21 articles were included in the study. The main risk factors found were: Work in critical care for patients with Covid-19, Use of biosafety equipment, Hospital infrastructure; Inadequate provision of Personal Protective Equipment, Age, gender, marital status, specialty and proximity to Covid-19 patients; Impossibility to meet the psycho-social-emotional needs of patients and families; Difficulty in externalizing your emotions; Lack of professional experience and Fear of contracting the virus and infecting others. The main risk factors for the outcomes mentioned above were: Emotional exhaustion; Psychological stress; anxiety; insomnia; fear; anger and frustration. Conclusions: The nurses working in the ICU during the Covid-19 pandemic presented psychiatric disorders, with emotional stress as the main causal factors, generated by structural and organizational inadequacies in health institutions.
\end{abstract}

Keywords: SARS-CoV-2; Intensive Therapy Units; Health care; Nursing care; Mental health.

\title{
Resumen
}

Introducción: Las enfermeras de primera línea enfrentan una enorme carga de trabajo, fatiga a largo plazo, amenaza de infección y frustración con la muerte de los pacientes que atienden. Objetivo: Investigar la evidencia actual sobre la aparición de condiciones psiquiátricas en enfermeras que trabajan en Unidades de Cuidados Intensivos durante la pandemia de Covid-19. Metodología: Estudio de revisión integrativa con búsqueda sistemática, realizado en las bases de datos PubMed, Embase, Cochrane Wiley y Virtual Health Library (VHL). Los criterios de inclusión fueron: (1) artículos electrónicos, (2) disponibles en su totalidad, (3) en portugués, inglés y / o español. Se excluyeron resúmenes, cartas al editor y estudios no relacionados con el tema. Resultados: Después de aplicar los criterios de elegibilidad, se incluyeron 21 artículos en el estudio. Los principales factores de riesgo encontrados fueron: Trabajo en cuidados críticos para pacientes con Covid-19, Uso de equipos de bioseguridad, Infraestructura hospitalaria; Provisión inadecuada de equipo de protección personal, edad, sexo, estado civil, especialidad y proximidad a los pacientes con Covid-19; Imposibilidad de satisfacer las necesidades psico-social-emocionales de los pacientes y sus familias; Dificultad para exteriorizar sus emociones; Falta de experiencia profesional y Miedo a contraer el virus e infectar a otros. Los principales factores de riesgo de los resultados mencionados anteriormente fueron: agotamiento emocional; Estrés psicológico; ansiedad; insomnio; temor; ira y frustración. Conclusiones: Los enfermeros que laboraron en la UCI durante la pandemia de Covid-19 presentaron trastornos psiquiátricos, siendo el estrés emocional el principal factor causal, generado por deficiencias estructurales y organizacionales en las instituciones de salud.

Palabras clave: SARS-CoV-2; Unidades de Cuidados Intensivos; Cuidado de la salud; Cuidado de enfermera; Salud mental.

\section{Introdução}

A Covid-19 é uma infecção de rápida disseminação, iniciada na China, no início de dezembro de 2019. A princípio denominada 2019-nCoV pela Organização Mundial da Saúde (OMS). Mais recentemente, passou a ser chamado de SARSCoV-2 (do inglês Severe Acute Respiratory Syndrome Coronavirus). A Covid-19 provocou profundo impacto global, sendo considerada a síndrome respiratória viral mais severa desde a pandemia de influenza H1N1, em 1918 (Ferguson et al, 2020). Os sintomas físicos da Covid-19 frequentemente envolvem febre, fadiga e tosse seca, podendo evoluir para dispneia ou, em casos mais graves, Síndrome Respiratória Aguda Grave (SRAG) que podem levar à morte (Brasil, 2020). 
O significativo número de casos que demandam internação hospitalar, incluindo cuidados em unidade de terapia intensiva, bem como a ausência de intervenções farmacológicas eficazes e seguras, tais como medicamentos ou vacinas, têm gerado preocupações sobre o colapso do sistema de saúde em diferentes nações (Ferguson et al., 2020).

A pandemia mudou a rotina de diversas pessoas e profissionais, impondo o isolamento social e/ou intensificação da carga de trabalho como uma medida para a contensão do vírus. No entanto, essa necessidade contribuiu para o desenvolvimento ou agravamento dos distúrbios mentais (Freire et al., 2021). Diante desse cenário, emerge paralelamente os problemas de saúde mental e o sofrimento psíquico, no âmbito dos espaços de trabalho na área da saúde.

O aumento súbito da demanda por assistência imediata, sobrecarrega todos os níveis de atenção, especialmente o terciário (nível hospitalar), desencadeando uma elevação da demanda de trabalho (Mo et al., 2020). Soma-se a isso, a oferta inadequada de Equipamentos de Proteção Individual (EPI’S) na maioria dos serviços, o medo de contrair a doença e por, consequentemente, contaminar seus familiares, são importantes fatores que somados aos já existentes, fora dos espaços de trabalho, favorecem o surgimento de problemas de saúde mental dos trabalhadores da área da saúde que atuam no cuidado aos pacientes portadores da Covid-19.

Os profissionais de enfermagem são protagonistas em espaços de cuidados em saúde. Não obstante, o contexto da pandemia tem evidenciado e reafirmado ainda mais esse protagonismo. No Brasil e no mundo, a equipe de enfermagem está na linha de frente contra o Coronavírus e tem um papel importante em todos os níveis de atenção, tornando sua presença indispensável no combate da Covid-19 e no atendimento aos pacientes infectados. Esses profissionais representam maioria nos serviços públicos e privados, sendo essenciais e considerados nucleares na estrutura das profissões da saúde (Duarte et al., 2021)

A enfermagem está na linha de frente dos atendimentos nos casos de Covid-19, sendo os únicos que permanecem 24 horas ao lado do paciente, estando, portanto, mais susceptíveis à infecção pelo novo Coronavírus e a desencadearem transtornos de ordem emocional em decorrência do trabalho (Souza e Souza \& de Souza, 2020). Os enfermeiros da linha de frente enfrentam uma enorme carga de trabalho, fadiga de longo prazo, ameaça de infecção e frustração com a morte de pacientes de quem cuidam. À medida que a pandemia avança, os sistemas de saúde ficam sobrecarregados gerando uma sobrecarga ainda maior junto aos enfermeiros de Unidades de Terapia Intensiva, levando a uma grande pressão psicológica sobre esses profissionais no cuidado de pacientes gravemente enfermos com Covid-19. Diante desse cenário, este estudo teve como objetivo investigar as atuais evidências sobre o surgimento de quadros psiquiátricos em enfermeiros de Unidades de Terapia Intensiva atuantes durante a pandemia da Covid-19.

\section{Metodologia}

Trata-se de uma revisão de literatura integrativa, a qual busca abordar lacunas de temas específicos, a partir da síntese de múltiplos estudos, permitindo uma discussão abrangente sobre os métodos, resultados e conclusões dos manuscritos coletados. Nesse cenário, este estudo baseou-se na pergunta norteadora: como o contexto da pandemia da Covid-19 vem refletindo no surgimento de quadros de ansiedade em Enfermeiros de Unidades de Terapia Intensiva?

Os procedimentos metodológicos desse estudo foram delineados de acordo com Chrizostimo e Caldas (2021). Foi realizada uma busca sistemática nas bases de dados: Medline (PubMed), Embase, Cochrane Wiley, e Biblioteca Virtual em Saúde (BVS). Os critérios de inclusão para coleta dos manuscritos foram: (1) artigos eletrônicos, (2) disponíveis na íntegra, (3) nos idiomas português, inglês e/ou espanhol. Foram excluídos artigos do tipo resumos, carta ao editor, manuscritos duplicados e estudos que não apresentaram relação com a temática. Ademais, a busca sistemática foi realizada nos meses de março e abril de 2021 e não considerou a literatura cinzenta.

Com base nos vocabulários controlados para indexação de artigos nas plataformas Mesh, Entree e Descritores em 
Ciências da Saúde (DeCS), foram definidos os descritores: "COVID-19", "Critical Care Nursing", "Nursing Critical Care", “coronavirus disease 2019”, "Intensive care nursing”. Posteriormente, utilizou-se a ferramenta de pesquisa avançada das bases de dados supracitada para a realização da estratégia de busca sistemática. Tais descritores foram combinados, entre si, utilizando o operador booleano AND para fazer a intersecção entre as linhas da estratégia de busca, resultando nas seguintes combinações: (1) COVID-19 AND Critical Care Nursing; (2) COVID-19 AND Nursing Critical Care; (3) "coronavirus disease 2019” AND "intensive care nursing”. Essas combinações foram rastreadas pelo título, resumo e assunto dos manuscritos, resultando em um total de 1.381 artigos (Figura 1).

Figura 1. Fluxograma da seleção e elegibilidade dos artigos.

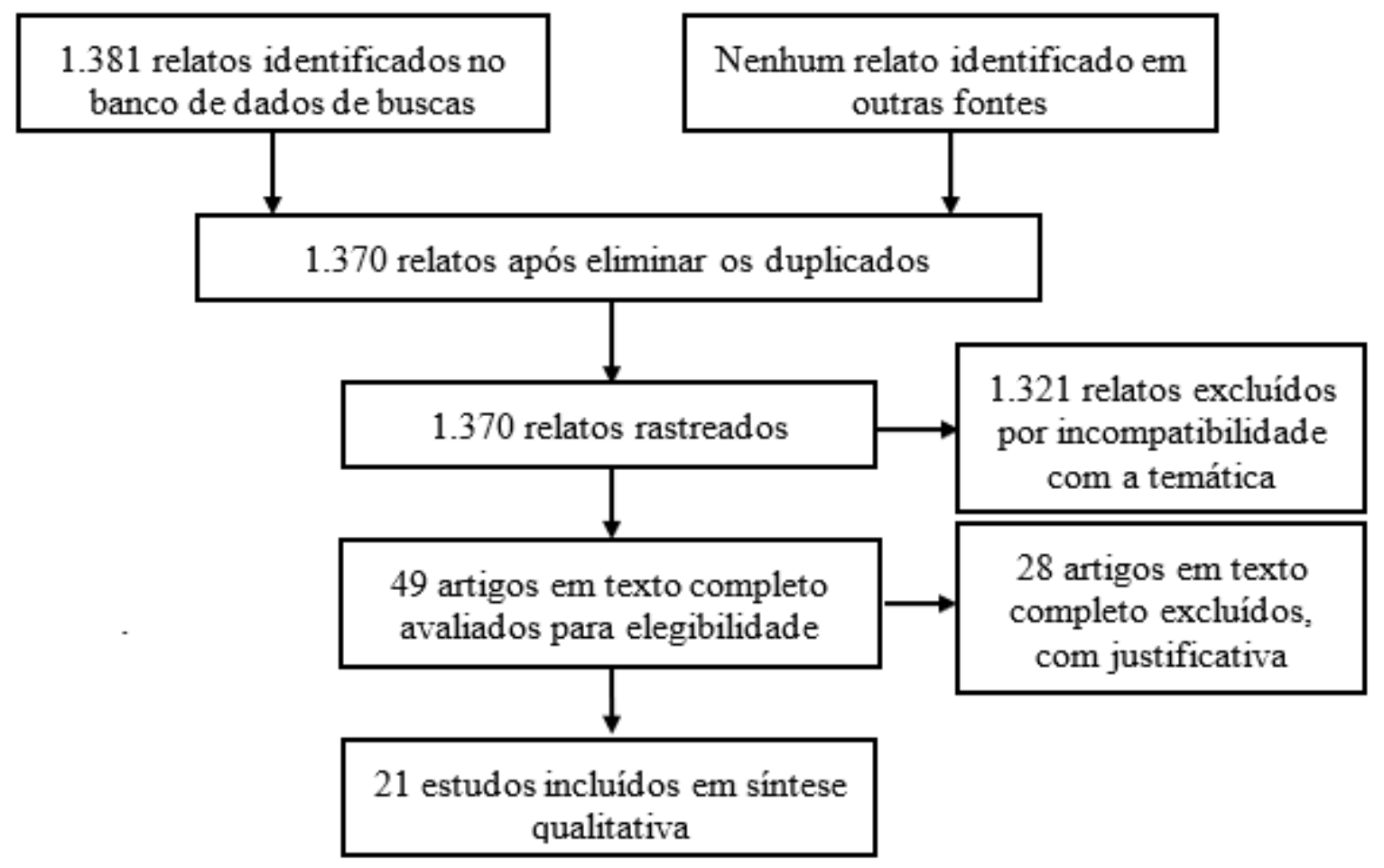

Fonte: Autores.

Foram identificadas, inicialmente, 1.381 publicações potencialmente elegíveis para este manuscrito. Após a primeira análise, com avaliação dos títulos e temática, 49 artigos foram considerados para a segunda fase. Na segunda fase, realizou-se a leitura dos resumos, os estudos que preencheram os critérios de inclusão foram lidos na íntegra. Ao final, 21 artigos (Embase 1 artigos), (Cochrane - 0 artigos), (MedLine - 19 artigos), (BVS - 1 artigo), atenderam a todos os critérios e subsidiaram a presente revisão.

Foram extraídos os seguintes dados dos artigos selecionados: Ano e local de realização do estudo, público investigado, principais fatores de risco e desfechos emocionais. Estes trabalhos foram organizados em tabelas e analisados de acordo com as características em comum de desfecho e fatores de risco associados.

\section{Resultados}

A maioria dos estudos seguiu um delineamento metodológico com análise qualitativa ( $n=17)$, baseada nas respostas dos sujeitos pesquisados. Os principais instrumentos de coleta de dados utilizados nos estudos foram questionários online. Os 
dados de caracterização dos artigos selecionados serão expostos no Quadro 1.

Quadro 1. Caracterização sistemática dos estudos.

\begin{tabular}{|l|l|l|}
\hline \multicolumn{1}{|c|}{ ESTUDO } & \multicolumn{1}{|c|}{ LOCAL DO ESTUDO } & \multicolumn{1}{c|}{ TIPO DE ESTUDO } \\
\hline 1. Vitale E. (2020) & 1. Itália & 1. Quantitativo \\
2. Bates A. (2020) & 2. Alemanha & 2. Quantitativo \\
3. Carrasco, O.P.C. et al (2020) & 3. China & 3. Quantitativo \\
4. Bohlken, L. et al (2020) & 4. Alemanha & 4. Revisão de Literatura \\
5. Shen, X. et al (2020) & 5. China & 5. Relato de Experiência \\
6. González-Gil, M. T. et al (2020) & 6. Espanha & 6. Qualitativo \\
7. Reignier. et al (2020) & 7. França & 7. Quantitativo \\
8. Chen, R. et al (2021) & 8. China & 8. Quantitativo \\
9. Xie, H. et al (2019) & 9. China & 9. Quantitativo \\
10. Zheng, R. et al (2020) & 10. China & 10. Quantitativo \\
11. Min Leng M.S.N. et al (2020) & 11. China & 11. Quantitativo \\
12. Crowe, S. et al (2020) & 12. Canadá & 12. Quantitativo \\
13. Altmayer, V. et al (2021) & 13. Paris & 13. Quantitativo e Qualitativo \\
14. Lapum, L. et al (2020) & 14. Canadá & 14. Qualitativo \\
15. Karabulut, N. et al (2020) & 15. Turquia & 15. Quantitativo \\
16. Carroll, R. F. et al (2021) & 16. Irlanda & 16. Quantitativo e Qualitativo \\
17. Ide, K. et al (2021) & 17. Japão & 17. Qualitativo \\
18. Jafarabadi, M. A. et al (2021) & 18. Irã & 18. Quantitativo \\
19. Vitale, E. et al (2020) & 19. Estados Unidos & 19. Quantitativo e Qualitativo \\
20. Bahadir, E. et al (2020) & 20. Turqui & 20. Qualitativo \\
21. Feeley, T. et al (2021) & 21. Irlanda & 21. Quantitativo e Qualitativo \\
\hline
\end{tabular}

Fonte: Autores.

A literatura científica aponta diversos fatores no desencadeamento de episódios de desordem emocionais entre enfermeiros, dentre eles, o perfil do paciente ao qual a assistência é prestada. A alta gravidade, e velocidade de deterioração da doença Covid-19 e o alto grau de transmissividade. A necessidade do uso de EPIs, por tempo prolongado. Além do medo de contrair a doença e contaminar seus familiares, foram relatos bastante prevalentes entre os sujeitos investigados.

Questões de ordem estrutural, como falta de suprimentos de insumos (EPIs), infraestrutura hospitalar e falta de experiência profissional também foram relatadas como importantes pontos geradores dos mais diversos quadros de depressão, ansiedade, estresse emocional e psicológico, além de medo, raiva e frustração.

A seguir serão apresentados os fatores de risco para os transtornos psiquiátricos e suas respectivas manifestações psicoemocionais (Quadro 2). 
Quadro 2. Distribuição dos fatores de risco e os desfechos dos transtornos de ordem emocional observados entre os enfermeiros de Unidades de Terapia Intensiva durante a pandemia da Covid-19, 2021.

\begin{tabular}{|c|c|c|}
\hline AUTOR & FATORES DE RISCO & DESFECHO EMOCIONAL \\
\hline $\begin{array}{l}\text { Vitale E. (2020) (a) } \\
\text { Shen, X. et al (2020) } \\
\text { Xie, H. et al (2019) } \\
\text { Crowe, S. et al (2020) } \\
\text { Altmayer, V. et al (2021) } \\
\text { Feeley, T. et al (2021) }\end{array}$ & $\begin{array}{l}\text { Trabalho em cuidados críticos } \\
\text { voltados para pacientes com Covid- } 19 .\end{array}$ & Exaustão Emocional. \\
\hline Bates A. (2020) & $\begin{array}{l}\text { Uso de equipamentos de } \\
\text { biossegurança por } 12 \text { horas contínuas; } \\
\text { Progressão da deterioração de } \\
\text { pacientes infectados com COVID }\end{array}$ & Estresse Psicológico. \\
\hline $\begin{array}{l}\text { Carrasco, O.P.C. et al (2020) } \\
\text { Reignier, E. et al (2020) } \\
\text { Jafarabadi, M. A. et al (2021) } \\
\text { Vitale, E. et al (2020) (b) } \\
\text { Bahadir, E. et al (2020) } \\
\text { Feeley, T. et al (2021) }\end{array}$ & $\begin{array}{l}\text { Infraestrutura hospitalar; } \\
\text { Fornecimento inadequado de } \\
\text { Equipamentos de Proteção Individual. }\end{array}$ & Estresse Psicológico \\
\hline $\begin{array}{l}\text { Bohlken, L. et al (2020) } \\
\text { Karabulut, N. et al (2020) } \\
\text { Jafarabadi, M. A. et al (2021) } \\
\text { Feeley, T. et al (2021) }\end{array}$ & $\begin{array}{l}\text { Idade, gênero, } \\
\text { estado civil, } \\
\text { especialidade, tipo de atividade e } \\
\text { proximidade com os pacientes } \\
\text { COVID-19. }\end{array}$ & Estresse, depressão e ansiedade \\
\hline $\begin{array}{l}\text { González-Gil, M. T. et al (2020) } \\
\text { Zheng, R. et al (2020) } \\
\text { Ide, K. et al (2021) }\end{array}$ & $\begin{array}{l}\text { Impossibilidade de atender às } \\
\text { necessidades psico-socioemocionais } \\
\text { dos pacientes e familiares; Dificuldade } \\
\text { de desabafar suas emoções. }\end{array}$ & $\begin{array}{l}\text { Ansiedade, depressão, insônia e } \\
\text { estresse psicológico. }\end{array}$ \\
\hline $\begin{array}{l}\text { Min Leng M.S.N. et al } \\
(2020) \\
\text { Carroll, R. F. et al (2021) } \\
\text { Jafarabadi, M. A. et al (2021) }\end{array}$ & Falta de experiência profissional. & Estresse emocional. \\
\hline $\begin{array}{l}\text { Lapum, L. et al (2020) } \\
\text { Bahadir, E. et al (2020) }\end{array}$ & $\begin{array}{l}\text { Medo de contrair o vírus e infectar } \\
\text { outras pessoas. }\end{array}$ & $\begin{array}{l}\text { Medo, isolamento, raiva } \mathrm{e} \\
\text { frustração. }\end{array}$ \\
\hline
\end{tabular}

Fonte: Autores.

\section{Discussão}

De acordo com os estudos coletados nesta revisão, as principais manifestações de ordem emocional percebidas em enfermeiras que atuam em Unidades de Terapia Intensiva - UTIs, são inerentes a configuração do trabalho que realizam. Essas manifestações são atribuídas ao contexto de vulnerabilidade inerente a pandemia da Covid-19 em diferentes países do cenário mundial.

Trabalhar em meio a uma pandemia exige que os profissionais e serviços de saúde tenham uma estrutura forte, capaz de comandar e controlar a tomada de decisões e informações, permitindo o enfrentamento a pandemia da melhor maneira possível. Profissionais de todo o mundo vivenciam exaustivos turnos de trabalho, atuando tanto no atendimento aos casos mais complexos quanto na prevenção à doença (Choi et al., 2020).

Diversos estudos apontam que os profissionais atuantes na linha de frente da covid-19, principalmente os que lidam diretamente com os cuidados a pacientes críticos, apresentaram variados desfechos de ordem emocional, tendo como principal quadro resultante do excessivo desgaste mental a exaustão emocional (Altmayer et al., 2020; Crowe et al., 2020; Feeley et al., 2021; Shen X et al., 2020; Vitale et al., 2020; Xie et al., 2020).

Os profissionais de saúde, destacando os enfermeiros, atuam na linha de frente, diante de uma jornada extenuante de trabalho e, sob risco elevado de contaminação, como por exemplo, ao manusear secreções dos pacientes. Esses profissionais 
são os mais próximos dos pacientes acometidos pela covid-19, estando ao seu lado durante maior parte do tempo de internação (Duarte et al., 2021). Por esse motivo, o enfermeiro assume o espaço de maior exposição e riscos à saúde em decorrência do trabalho que executam junto aos pacientes, levando-os a situações de vulnerabilidade não somente física, como também emocional.

A progressão crítica dos pacientes infectados com Covid-19, bem como o uso de equipamentos de biossegurança por 12 horas contínuas, é apontada como os principais fatores diretamente associados aos quadros de exaustão emocional e transtornos psicológicos entre profissionais da saúde, principalmente enfermeiros atuantes na UTI (2020).

Os equipamentos de biossegurança estão relacionados a uma elevação incidência de complicações cutâneas. Esse fato pode levar a descontinuação do uso dos EPIs por parte dos profissionais. Além disso, o uso prolongado desses equipamentos pode dificultar na frequência da hidratação e de esvaziamento vesical dos profissionais de saúde, favorecendo o surgimento de complicações de saúde (Koh, 2020).

Diante da realidade conflitante em que se deparam os profissionais de cuidados a pacientes graves em decorrência da Covid-19, as situações de morte e estresse vivenciados são constantes, tornando os ambientes assistenciais, muitas vezes, sobrecarregados emocionalmente. A necessidade de um atendimento de enfermagem preciso e cauteloso, tanto nos procedimentos técnicos quanto na paramentação e desparamentação rigorosa, conforme recomendado cientificamente, também são aspectos que contribuem para a exaustão (Jackson et al., 2020; Miranda et al., 2020).

Estudos apontam a infraestrutura hospitalar e o fornecimento inadequado de Equipamentos de Proteção Individual (EPIs) para os profissionais de saúde que atuam em cuidados críticos na Covid-19, como fatores responsáveis pelo surgimento dos transtornos de estresse psicológico (Azoulay et al., 2020; Bahadir-Yilmaz \& Yüksel, 2020; Carrasco et al., 2020; Feeley et al., 2021; Jafarabadi et al., 2021; Vitale et al., 2020). Enfermeiros de diferentes regiões do mundo e lutam contra a escassez de suprimentos, incluindo equipamentos de proteção individual, como máscaras, luvas e aventais, além da luta pela melhoria na reestruturação dos serviços (Jackson et al., 2020).

Ademais, idade, gênero, estado civil, especialidade, tipo de atividade e proximidade com os pacientes com Covid-19 são também destacados na literatura científica como fatores potenciais para o estresse psicológicos (Bohlken et al., 2020; Carroll et al., 2020; Jafarabadi et al., 2021; Karabulut et al., 2021). A idade avançada, enfermeiros que não possuíam companheiros, bem como aqueles que foram realocados para os setores críticos, sem a devida especialização/experiência para atuarem nesses espaços, favoreceram ao surgimento de quadros de estresse, depressão e ansiedade entre os profissionais de enfermagem investigados.

A heterogeneidade que caracteriza esse contingente da força de trabalho determina formas diferentes de exposição, tanto ao risco de contaminação quanto aos fatores associados às condições de trabalho, podendo levar a quadros de transtornos psicológicos dentre eles, estresse, ansiedade e depressão (Teixeira et al., 2020). Além disso, a impossibilidade de atender às necessidades psico-socioemocionais dos pacientes e familiares e a dificuldade de expressar suas emoções, são catalizadores do desenvolvimento de estresse, ansiedade e angústia (et al. González-gil, 2020; Ide et al., 2021; Zheng et al., 2020).

A escolha pela profissão "enfermagem" é motivada pelo desejo de ajudar as pessoas a recuperar e manter a saúde e, aqui, temos uma situação em que pode haver poucas opções para ajudar aqueles que estão gravemente doentes por causa da Covid-19. Assim, diante do cenário incerto, os enfermeiros se veem impossibilitados de prever um prognóstico positivo para seus pacientes, o que pode gerar o sentimento de tristeza e frustração, refletindo negativamente em seus estados emocionais (Jackson et al., 2020).

O sistema de apoio social do enfermeiro deve ser ativamente mobilizado. Este deve manter contato com seus familiares e amigos para obter apoio espiritual e emocional. Ao mesmo tempo, os enfermeiros devem encorajar uns aos outros, discutir e compartilhar sentimentos e experiências com seus colegas de maneira oportuna e desabafar emoções negativas. A 
adoção de condutas de coleguismo e cooperação mútua, cria um espaço de felicidade e de alívio de conflitos internalizados pelo trabalho físico e emocionalmente sobrecarregados (Mo et al., 2020).

A falta de experiência diante de uma patologia até então desconhecida, foi o principal motivo do estresse emocional entre os enfermeiros. Este fato sofrimento psicológico ao assumir papeis clínicos novos ou desconhecidos. O desconhecido causa medo e ansiedade em profissionais de qualquer área de atuação. Não obstante, quando o cenário envolve a saúde de seres humanos e, sobretudo, em um contexto hostil, esse sentimento torna-se ainda mais presente e intenso entre os profissionais da linha de frente. Gerando sentimentos de angústia, ansiedade e estresse emocional (Carroll et al., 2020; Jafarabadi et al., 2021).

O medo de contrair o vírus e infectar outras pessoas é outro ponto que contribui para o desgaste mental, desencadeando sentimentos como medo, isolamento, raiva e frustração (Bahadir-Yilmaz \& Yüksel, 2020; Lapum et al., 2021; Teixeira et al., 2020). Entre relatos profissionais, os pontos mais fortemente abordados são, o medo da contaminação e de contaminarem familiares e amigos. Sendo este um dos principais responsáveis pelo surgimento de estados emocionais adoecidos entre os sujeitos estudos.

Existe uma diversidade de questões que permeiam os espaços de produção em saúde voltados para pacientes críticos acometidos pela doença Covid-19. É válido apesar nas questões aqui analisadas. Ainda que estas sejam de ordem emocional e, por esse motivo, em algumas situações, entendidas como subjetivas, foi percebido que muitos desses fatores geradores de desordem emocional, podem ser minimizados, por meio de estratégias, ações, bem como pela restruturação dos espações produtores do cuidado e de assistência.

Poucos estudos abordaram o apontamento ou a apresentação de estratégias, bem como de medidas protetivas e ações estruturais voltadas para a preservação da saúde emocionais dos trabalhadores de saúde/enfermagem. É necessário e urgente o desenvolvimento de novos estudos que avancem em aspectos como, estratégias de resolução de conflitos no tange ao trabalho de reparação dos danos deixados por esse fenômeno devastador que está sendo a pandemia da Covid-19.

\section{Considerações Finais}

A literatura explorada no presente estudo revela que o contexto da pandemia da Covid-19 vem refletindo no surgimento de quadros de ansiedade em enfermeiros de Unidade de Terapia Intensiva.

Os achados científicos reafirmaram que a própria conformação do trabalho dos profissionais de saúde, gera uma inclinação ao surgimento de transtornos de ordem psicoemocionais. Observou-se que muitos trabalhadores da saúde, sobretudo os da enfermagem, trazem consigo intrinsecamente, algum tipo de desordem emocional, em diferentes níveis, alguns mais sutis e leves, outros mais evidentes.

Inúmeros fatores foram apontados como geradores de quadros de ansiedade entre os enfermeiros que atuam em UTI's voltadas para o tratamento da Covid-19. Porém existiram aqueles que mereceram posição de destaque nessa pesquisa. Dentre esses estão: o perfil do paciente acometido pelo vírus SARS-CoV-2, a falta de estruturação dos serviços, nos mais diversos aspectos. E o medo da contaminação e da propagação da doença para os familiares. Além da falta de experiência profissional existente em muitos enfermeiros.

As potencialidades observadas nesse artigo de revisão consistem na identificação dos fatores geradores de estresse emocionais entre os enfermeiros de UTIs que devem ser corrigidos por meio de medidas estruturais e organizacionais. Destacamos a necessidade de mais estudos e pesquisas que compartilhem experiências, bem como sugestões de estratégias que busquem tratar dos prejuízos psicológicos deixados por esse fenômeno devastador que é a pandemia da Covid-19.

Este estudo apresenta limitações que devem ser levadas em consideração. Em primeiro lugar, avalia a prevalência de sintomas de ansiedade, depressão, estresse psicológico e emocional em um determinado momento. Esses sintomas podem ser transitórios e são necessários estudos de acompanhamento de longo prazo. Este estudo, apesar de identificar a presença do 
sofrimento psíquico, não permite demonstrar que o Covid-19 foi responsável por sobrecarga psicológica adicional nos enfermeiros de Unidades de Terapia Intensiva, uma vez que não há dados pré- Covid-19 disponíveis. Faz-se necessária a realização de estudos com ferramentas capazes de mensurar essas características no período anterior a pandemia e avaliar as mudanças longitudinais ocorridas nos estados de saúde mental dos enfermeiros.

\section{Referências}

Altmayer, V., Weiss, N., Ph, D., Cao, A., Demeret, S., Rohaut, B., \& Ph, D. (2020). Since January 2020 Elsevier has created a COVID-19 resource centre with free information in English and Mandarin on the novel coronavirus COVID- 19. The COVID-19 resource centre is hosted on Elsevier Connect, the company' s public news and information. January.

Azoulay, E., Cariou, A., Bruneel, F., Demoule, A., Kouatchet, A., Reuter, D., Souppart, V., Combes, A., Klouche, K., Argaud, L., Barbier, F., Jourdain, M., Reignier, J., Papazian, L., Guidet, B., Geri, G., Resche-Rigon, M., Guisset, O., Labbe, V., \& Kentish-Barnes, N. (2020). Symptoms of anxiety, depression, and peritraumatic dissociation in critical care clinicians managing patients with COVID-19 a cross-sectional study. Am. J. Respir. Crit. Care Med., 202(10), 13881398. https://doi.org/10.1164/rccm.202006-25680C

Bahadir-Yilmaz, E., \& Yüksel, A. (2020). State anxiety levels of nurses providing care to patients with COVID-19 in Turkey. Perspect. Psychiatr. Care, October. https://doi.org/10.1111/ppc.12661

Bates, A., Ottaway, J., Moyses, H., Perrow, M., Rushbrook, S., \& Cusack, R. (2020). Psychological impact of caring for critically ill patients during the Covid-19 pandemic and recommendations for staff support. J. Intensive Care Soc., 0(0), 1-7. https://doi.org/10.1177/1751143720965109

Bohlken, J., Schömig, F., Lemke, M. R., Pumberger, M., \& Riedel-Heller, S. G. (2020). COVID-19-Pandemie: Belastungen des medizinischen Personals. Psychiatr. Prax., 47(04), 190-197. https://doi.org/10.1055/a-1159-5551

Brasil, M. da S. (2020). Diagnóstico e Tratamento da COVID-19 TRATAMENTO. Secretaria De Ciência, Tecnologia, Inovação E Insumos Estratégicos Em Saúde, 1, 1-398.

Carrasco, O., Castillo, E., Salas, R., \& Reyes, C. (2020). Estresores laborales y satisfacción en enfermeras peruanas durante la pandemia de COVID - 19. Scielo Prepr., 1(1), 1-14. https://preprints.scielo.org/index.php/scielo/preprint/view/1468/2323

Carroll, R., Feeley, T., Tan, M. H., Magner, C., Estrange, K. L., Efrimescu, C., Connor, E. O., Lyons, B., \& Crowe, S. (2020). Since January 2020 Elsevier has created a COVID-19 resource centre with free information in English and Mandarin on the novel coronavirus COVID- 19. The COVID-19 resource centre is hosted on Elsevier Connect, the company 's public news and information. January. https://doi.org/10.1016/j.bja.2020.09.0

Choi, K. R., Skrine Jeffers, K., \& Cynthia Logsdon, M. (2020). Nursing and the novel coronavirus: Risks and responsibilities in a global outbreak. J. Adv. Nurs., 76(7), 1486-1487. https://doi.org/10.1111/jan.14369

Chrizostimo, M. M. (2021). Formação profissional de enfermeiro gerontológico : revisão integrativa Professional training of gerontological nurses : integrative review Formación profesional de enfermeras gerontológicas : revisión integradora. 2021, 1-13.

Crowe, S., Howard, A. F., Vanderspank-wright, B., Gillis, P., \& Mcleod, F. (2020). Since January 2020 Elsevier has created a COVID-19 resource centre with free information in English and Mandarin on the novel coronavirus COVID- 19. The COVID-19 resource centre is hosted on Elsevier Connect, the company' s public news and information. January.

Duarte, M., Silva, D., \& Bagatini, M. (2021). Enfermagem e saúde mental: uma reflexão em meio à pandemia do coronavírus. Rev. Gaúcha Enferm., 42.

Feeley, T., Ffrench-O'Carroll, R., Tan, M. H., Magner, C., L’Estrange, K., O’Rathallaigh, E., Whelan, S., Lyons, B., \& O’Connor, E. (2021). A model for occupational stress amongst paediatric and adult critical care staff during COVID-19 pandemic. Int. Arch. Occup. Environ. Health. https://doi.org/10.1007/s00420-021-01670-6

Ferguson, N. M., Laydon, D., Nedjati-Gilani, G., Imai, N., Ainslie, K., Baguelin, M., Bhatia, S., Boonyasiri, A., Cucunubá, Z., Cuomo-Dannenburg, G., \& Dighe, A. (2020). Report 9 - Impact of non-pharmaceutical interventions (NPIs) to reduce COVID-19 mortality and healthcare demand | Faculty of Medicine | Imperial College London. Imp. Coll. COVID Response Team, March, 20. https://doi.org/10.25561/77482

Freire, L. F. de O., Cursino, M. A., Leal, D. C. L., Morais, A. de F., Regis, M. da S., Lima, I. P. C., Leal, R. M. S., \& Silva, M. G. da. (2021). Pandemia COVID-19: efeitos do distanciamento social no comportamento alimentar de estudantes do estado do Ceará. Research, Society and Development, 10(11), e47101119185. https://doi.org/10.33448/rsd-v10i11.19185

González-Gil, M. T. et al. (2020). Since January 2020 Elsevier has created a COVID-19 resource centre with free information in English and Mandarin on the novel coronavirus COVID- 19. The COVID-19 resource centre is hosted on Elsevier Connect, the company's public news and information. Intensive Crit. Care Nurs., January. https://doi.org/10.1016/j.iccn.2020.102966

Ide, K., Asami, T., Suda, A., Yoshimi, A., Fujita, J., Nomoto, M., Roppongi, T., Hino, K., Takahashi, Y., Watanabe, K., Shimada, T., Hamasaki, T., Endo, E., Kaneko, T., Suzuki, M., Kubota, K., Saigusa, Y., Kato, H., Odawara, T., ... Hishimoto, A. (2021). The psychological effects of COVID-19 on hospital workers at the beginning of the outbreak with a large disease cluster on the Diamond Princess cruise ship. PLoS One, $16(1$ January), 1-15. https://doi.org/10.1371/journal.pone.0245294

Jackson, D., Bradbury-Jones, C., Baptiste, D., Gelling, L., Morin, K., Neville, S., \& Smith, G. D. (2020). Life in the pandemic: Some reflections on nursing in the context of COVID-19. J. Clin. Nurs., 29(13-14), 2041-2043. https://doi.org/10.1111/jocn.15257 
Research, Society and Development, v. 10, n. 15, e125101522749, 2021

(CC BY 4.0) | ISSN 2525-3409 | DOI: http://dx.doi.org/10.33448/rsd-v10i15.22749

Jafarabadi, M. A., Vahedian-Azimi, A., Rahimibashar, F., Guest, P. C., Karimi, L., \& Sahebkar, A. (2021). Psychometric Evaluation of Stress in 17,414 Critical Care Unit Nurses: Effects of Age, Gender, and Working Conditions. Adv. Exp. Med. Biol., 1286, 199-212. https://doi.org/10.1007/978-3-030-55035$6 \_14$

Karabulut, N., Gürçayır, D., Yaman Aktaş, Y., Kara, A., Kızıloğlu, B., Arslan, B., \& Bölükbaş, N. (2021). The effect of perceived stress on anxiety and sleep quality among healthcare professionals in intensive care units during the coronavirus pandemic. Psychol. Heal. Med., 26(1), 119-130. https://doi.org/10.1080/13548506.2020.1856897

Koh, D. (2020). Occupational risks for COVID-19 infection. Occup. Med. (Chic. Ill)., 70(1), 3-5. https://doi.org/10.1093/occmed/kqaa036

Lapum, J., Nguyen, M., Fredericks, S., Lai, S., \& McShane, J. (2021). "Goodbye ...Through a Glass Door”: Emotional Experiences of Working in COVID-19 Acute Care Hospital Environments. Can. J. Nurs. Res., 53(1), 5-15. https://doi.org/10.1177/0844562120982420

Miranda, F. M. D., Santana, L. de L., Pizzolato, A. C., \& Saquis, L. M. M. (2020). Working conditions and the impact on the health of the nursing professionals in the context of covid-19. Cogitare Enferm., 25, 1-8. https://doi.org/10.5380/ce.v25i0.72702

Mo, Y., Deng, L., Zhang, L., Lang, Q., Liao, C., Wang, N., Qin, M., \& Huang, H. (2020). Work stress among Chinese nurses to support Wuhan in fighting against COVID-19 epidemic. J. Nurs. Manag., 28(5), 1002-1009. https://doi.org/10.1111/jonm.13014

Shen X, Zou X, Zhong X, Yan J, \& Li L. (2020). Psychological stress of ICU nurses in the time of COVID-19. Critical care[revista en internet] 2020 [acceso 09 de junio del 2020]; 24(1): 200. Crit. Care, 2-4. https://ccforum.biomedcentral.com/track/pdf/10.1186/s13054-020-02926-2

Souza e Souza, L. P., \& de Souza, A. G. (2020). Enfermagem brasileira na linha de frente contra o novo Coronavírus: quem cuidará de quem cuida? J. Nurs. Heal., 10(4). https://doi.org/10.15210/jonah.v10i4.18444

Teixeira, C. F. de S., Soares, C. M., Souza, E. A., Lisboa, E. S., Pinto, I. C. de M., de Andrade, L. R., \& Espiridião, M. A. (2020). The health of healthcare professionals coping with the covid-19 pandemic. Cienc. e Saude Coletiva, 25(9), 3465-3474. https://doi.org/10.1590/1413-81232020259.19562020

Vitale, E., Galatola, V., \& Mea, R. (2020). Exploring within and between gender differences in burnout levels in Italian nurses engaged in the cOviD-19 health emergency: A cohort observational study. Minerva Psichiatr., 61(4), 162-170. https://doi.org/10.23736/S0391-1772.20.02090-7

Xie, H., Cheng, X., Song, X., Wu, W., Chen, J., Xi, Z., \& Shou, K. (2020). Investigation of the Psychological disorders in the healthcare nurses during a coronavirus disease 2019 outbreak in China. Medicine (Baltimore)., 99(34), e21662. https://doi.org/10.1097/MD.0000000000021662

Zheng, R., Zhou, Y., Fu, Y., Xiang, Q., Cheng, F., \& Chen, H. (2020). Since January 2020 Elsevier has created a COVID-19 resource centre with free information in English and Mandarin on the novel coronavirus COVID- 19. The COVID-19 resource centre is hosted on Elsevier Connect, the company's public news and information. January. 